\title{
Características clínicas, patológicas y supervivencia de cáncer de mama no metastásico
}

\author{
- Pedro Luis Ramos Guette, María Athenas Ramos Escalante, Diana Silva, Mario Gonzáles, Mauricio García \\ Oncológica Oncocare (Bogotá, D.C.)
}

Introducción: el cáncer de mama es la primera causa de incidencia y segunda de mortalidad en mujeres en el mundo. El propósito de este estudio es describir las características clínicas, patológicas, y la supervivencia en cáncer de mama no metastásico.

Materiales y métodos: se trata de un estudio descriptivo retrospectivo efectuado durante el período marzo de 2013-septiembre de 2016. Se llevaron a cabo medidas descriptivas utilizando tendencia central, frecuencias absolutas y relativas. Se estimó la supervivencia por método de Kaplan-Meier.

Resultados: se identificó un total de 581 pacientes con un promedio de edad de 55,4 años. Estadios clínicos (I 11,19, IIA 16,87, IIB 18,76, IIIA 18,76, IIIB 24,27, IIIC 9,64) con enfermedad localmente avanzada en un $52,9 \%$. Ganglios positivos en un $70,05 \%$. Receptores hormonales positivos en un $74 \%$ y HER2 positivos del $18,9 \%$. En cuanto a los subtipos intrínsecos, el $74 \%$ son luminales y el $7 \%$ son triple negativos. Son posmenopáusicas el $63,51 \%$. Histología ductal el $88,6 \%$ y grado II el $53,87 \%$. En el $97,5 \%$ se realiza cirugía y en el $49,75 \%$, mastectomía. El $65 \%$ de los pacientes reciben quimioterapia neoadyuvante, el esquema más usado fue AC y ACT $(84,6 \%)$ y la hormonoterapia más frecuentemente utilizada fue el tamoxifen. Las respuestas completas patológicas fueron del 15,24\%. Las recaídas fueron de un $21 \%$ (local 4,65\%, regional 1,89 y sistémicas 19,79\%); los sitios más usuales de recaídas fueron óseos y en el pulmón; y la frecuencia de muertes específica fue de un 18,7\% y muertes por otras causas del 3,79\%.

Conclusiones: las características clínicas y patológicas y la supervivencia de los pacientes es comparable con las reportadas en la literatura, y la frecuencia de muerte es la esperada con una mediana de seguimiento de 93 meses. 